\title{
EXTENSION OF A THEOREM OF CARLESON
}

BY PETER L. DUREN

Communicated by John Wermer, July 29, 1968

One of the main ingredients in Carleson's solution to the corona problem [2] is the theorem characterizing the measures $\mu$ on the open unit disk with the property that $f \in H^{p}$ implies

$$
\int_{|z|<1}|f(z)|^{p} d \mu(z)<\infty, \quad 0<p<\infty .
$$

Carleson's proof of this theorem involves a difficult covering argument and the consideration of a certain quadratic form (see also [1]). L. Hörmander later found a proof which appeals to the Marcinkiewicz interpolation theorem and avoids any discussion of quadratic forms. The main difficulty in this approach is to show that a certain sublinear operator is of weak type $(1,1)$. Here a covering argument reappears which is similar to Carleson's but apparently easier (see [4]).

We wish to point out that Hörmander's argument, with appropriate modifications, actually proves the theorem in the following extended form.

THEOREM. Let $\mu$ be a finite measure on $|z|<1$, and suppose $0<p$ $\leqq q<\infty$. Then in order that there exist a constant $C$ such that

$$
\left\{\int_{|z|<1}|f(z)| q d \mu(z)\right\}^{1 / q} \leqq C\|f\|_{p}
$$

for all $f \in H^{p}$, it is necessary and sufficient that there be a constant $A$ such that

$$
\mu(S) \leqq A h^{q / p}
$$

for every set $S$ of the form

$$
S=\left\{\mathrm{re}^{i \theta}: 1-h \leqq r<1, \theta_{0} \leqq \theta \leqq \theta_{0}+h\right\} .
$$

Outline of Proof. A standard argument (factoring out Blaschke products) shows it is enough to consider the case $p=2$. The necessity of (2) is then proved by choosing $f(z)=(1-\alpha z)^{-1}$, where $|\alpha|<1$.

Conversely, let $p=2$ and suppose (2) holds. Since each $f \in H^{2}$ is the Poisson integral of its boundary function, it will be sufficient to prove that 


$$
\left\{\int_{|z|<1}[u(z)] q d \mu(z)\right\}^{1 / q} \leqq C\left\|_{\varphi}\right\|_{2}
$$

if $u(z)$ is the Poisson integral of a nonnegative function $\varphi \in L^{2}$.

With each point $z=\mathrm{re}^{i \theta}$ in $0<|z|<1$ we associate the boundary arc

$$
I_{z}=\left\{e^{i t}: \theta-\frac{1}{2}(1-r) \leqq t \leqq \theta+\frac{1}{2}(1-r)\right\} .
$$

Taking $0 \leqq \theta<2 \pi$, we can identify $I_{z}$ with a segment on the real line. Given an integrable function $\varphi(t) \geqq 0$, periodic with period $2 \pi$, define

$$
\tilde{\varphi}(z)=\sup \frac{1}{|I|} \int_{I} \varphi(t) d t
$$

where the supremum is taken over all intervals $I \supset I_{z}$ of length $|I|<1$. Then $\tilde{\varphi}(z)$ is continuous in $0<|z|<1$. It is not difficult to show that

$$
u(z) \leqq 16 \pi^{2}\left\{\tilde{\varphi}(z)+\|\varphi\|_{1}\right\}, \quad|z|<1,
$$

where $u$ is the Poisson integral of $\varphi$. Thus it will suffice to prove (4) with $\tilde{\varphi}$ replacing $u$.

In other words, we must show that the sublinear operator $T: \varphi \rightarrow \tilde{\varphi}$ is of type $(2, q)$. Since $T$ is trivially of type $(\infty, \infty)$, this will follow from the Marcinkiewicz interpolation theorem if it can be shown that $T$ is of weak type $(1, q)$ for $1 \leqq q<\infty$ :

$$
\mu\left(E_{8}\right) \leqq C s^{-q}\|\varphi\|_{1}^{q},
$$

where

$$
E_{s}=\{z: \tilde{\varphi}(z)>s\}, \quad s>0 .
$$

It is only in proving (5) that any use is made of the assumption that

$$
\mu(S) \leqq A h^{q}
$$

for all $S$ of the form (3). (For convenience, $q / 2$ has been replaced by $q$.)

Essentially following Hörmander [4], we define for each $\epsilon>0$ the sets

$$
A_{s}^{\mathrm{e}}=\left\{z: \int_{I_{z}}|\varphi(t)| d t>s\left(\epsilon+\left|I_{z}\right|\right)\right\}
$$

and

$$
B_{s}^{e}=\left\{z: I_{z} \subset I_{w} \text { for some } w \in A_{*}^{e}\right\}
$$


Note that

$$
\mu\left(E_{\imath}\right)=\lim _{\iota \rightarrow 0} \mu\left(B_{\imath}^{\bullet}\right)
$$

If $z_{n} \in A_{s}$ and the $\operatorname{arcs} I_{z_{n}}$ are disjoint, then

$$
s \sum_{n}\left(\epsilon+\left|I_{z_{n}}\right|\right)<\sum_{n} \int_{I_{z_{n}}}|\varphi(t)| d t \leqq 2 \pi\|\varphi\|_{1} .
$$

In particular, there can be at most a finite number of points $z_{n}$ in $A_{s}^{e}$ whose associated arcs $I_{z_{n}}$ are disjoint. The following lemma, whose proof we omit, is now needed (compare [4, Lemma 2.2]).

Covering Lemma. Let $A$ be a nonempty set in $|z|<1$ which contains no infinite sequence of points whose associated arcs $I_{z_{n}}$ are disjoint. Then there exists a finite number of points $z_{1}, \cdots, z_{m}$ in $A$ such that the arcs $I_{z_{n}}$ are disjoint and

$$
A \subset \bigcup_{n=1}^{m}\left\{z: I_{z} \subset J_{z_{n}}\right\}
$$

where $J_{z}$ is the arc of length $5\left|I_{z}\right|$ whose center coincides with that of $I_{z}$.

If $E_{s}$ is nonempty, the lemma gives (for some $\epsilon>0$ )

$$
A_{s}^{e} \subset \bigcup_{n=1}^{m}\left\{z: I_{z} \subset J_{z_{n}}\right\}
$$

where $z_{n} \in A_{\text {s }}^{\mathrm{s}}$ and the $\operatorname{arcs} I_{z_{n}}$ are disjoint. It follows that

$$
B_{s} \subset \bigcup_{n=1}^{m}\left\{z: I_{2} \subset J_{z_{n}}\right\}
$$

Thus the hypothesis (6) gives

$$
\mu\left(B_{s}^{e}\right) \leqq C \sum_{n=1}^{m}\left|I_{z_{n}}\right|^{q}
$$

But by (8) we have (since $q \geqq 1$ )

$$
\left\{\sum_{n=1}^{m}\left|I_{z_{n}}\right|^{q}\right\}^{1 / q} \leqq \sum_{n=1}^{m}\left|I_{z_{n}}\right|<2 \pi s^{-1}\|\varphi\|_{1} .
$$

This together with (7) and (9) proves (5), and (1) follows.

Two applications are worth noting: 
1. If $0<p<q<\infty$, then $f \in H^{p}$ implies

$$
\int_{0}^{1}(1-r)^{q / p-2} M_{q}^{q}(r, f) d r<\infty,
$$

where

$$
M_{q}^{q}(r, f)=\frac{1}{2 \pi} \int_{0}^{2 \pi}\left|f\left(\mathrm{re}^{i \theta}\right)\right|^{q} d \theta .
$$

This useful result is due to Hardy and Littlewood [3].

2. If $0<p \leqq q<\infty$, and $f \in H^{p}$, then

$$
\left\{\int_{-1}^{1}(1-r)^{q / p-1}|f(r)| q d r\right\}^{1 / q} \leqq C\|f\|_{p} .
$$

This is a generalization of the Fejér-Riesz theorem, aside from the value of the constant $C$.

\section{REFERENCES}

1. L. Carleson, An interpolation problem for bounded analytic functions, Amer. J. Math. 80 (1958), 921-930.

2. - Interpolations by bounded analytic functions and the corona problem, Ann. of Math. (2) 76 (1962), 547-559.

3. G. H. Hardy and J. E. Littlewood, Some properties of fractional integrals. II, Math. Z. 34 (1932), 403-439.

4. L. Hörmander, $L^{p}$ estimates for (pluri-) subharmonic functions, Math. Scand. 20 (1967), 65-78.

University of Michigan, ANn Arbor, Michigan 48104 and

Institute for Advanced Study, Princeton, New Jersey 08540 